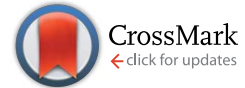

Cite this: RSC Adv., 2017, 7, 11170

Received 14th December 2016 Accepted 30th January 2017

DOI: 10.1039/c6ra28174e

rsc.li/rsc-advances

\section{Biomimetic surfaces with anisotropic sliding wetting by energy-modulation femtosecond laser irradiation for enhanced water collection $\uparrow$}

\author{
Yang Lu, ${ }^{a}$ Liandong Yu, ${ }^{a}$ Zhen Zhang, ${ }^{b}$ Sizhu Wu, ${ }^{* a}$ Guoqiang Li, ${ }^{\text {b }}$ Peichao Wu, ${ }^{b}$

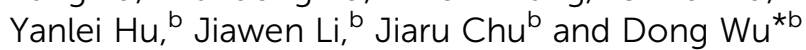

Biological rice leaf surfaces show a distinct anisotropic sliding property by means of three-level macrogrooves and micro/nanostructures, and they have many potential applications in biomimetic cell movement control, water transportation, and microfluidic devices. However, fabricating artificial threelevel biosurfaces with a controllable anisotropic sliding property by a simple and effective method remains a challenge. Herein, we report a simple method to prepare hierarchical groove structures (macro and micro/nano) on polydimethylsiloxane (PDMS) films using energy-modulation femtosecond laser scanning. The macrogrooves for anisotropic control were realized by larger-energy $\left(>0.40 \mathrm{~J} \mathrm{~cm}^{-2}\right)$ laser scanning, whereas the micro/nanostructures for superhydrophobic ability were fabricated by smallenergy $\left(0.08 \mathrm{~J} \mathrm{~cm}^{-2}\right)$ laser scanning. The processed surface shows a sliding angle (SA) difference of $6^{\circ}$ between the perpendicular and parallel directions, which is comparable to that of the natural rice leaf. To quantitatively investigate the anisotropic wettability, surfaces with a different period (100 to $600 \mu \mathrm{m}$ ) and height (30 to $100 \mu \mathrm{m}$ ) were systematically fabricated by adjusting the scanning space and pulse energy. Finally, the distinct ability of the dynamic water droplet anisotropic sliding and size-constrained fog deposition on the anisotropic biosurfaces was demonstrated. The collection efficiency of water on the anisotropic surface with a rotation of 5 and 10 degrees is four times and eighty times higher than that on an isotropic surface.

\section{Introduction}

As is well-known that butterflies can shed water from their body's center-axis, ${ }^{1}$ water birds let drops slide along the direction of their feather arrangement, ${ }^{2}$ and rice leaves guide the micro-droplets to trickle down in a direction parallel to the edge of the leaf. ${ }^{3}$ These natural characteristics of organisms have attracted scientific attention in bionic processing research ${ }^{4-7}$ and have inspired scientists to realize artificial anisotropic biosurfaces for enormous potential applications in microfluidic devices and liquid micro-droplet directional transfer. ${ }^{8-27}$ Among these, the surface wettability of rice leaves has been widely studied. ${ }^{9-15}$

Droplets on rice leaves can easily roll off. The sliding angles along the parallel and perpendicular direction are different,

${ }^{a}$ Precision and Equipment Support Laboratory, Department of Instrument Science \& Opto-electronics Engineering, Hefei University of Technology, Hefei, Anhui, 230009, People's Republic of China. E-mail: sizhuwu@hfut.edu.cn

${ }^{b}$ Department of Precision Machinery and Precision Instrumentation, University of Science and Technology of China, Hefei, Anhui, 230026, People's Republic of China. E-mail:dongwu@ustc.edu.cn

$\uparrow$ Electronic supplementary information (ESI) available. See DOI: $10.1039 / \mathrm{c} 6 \mathrm{ra} 28174 \mathrm{e}$ about $3^{\circ}$ and $9^{\circ}$, respectively. ${ }^{3}$ With this property, the rice roots easily get the water rolling down from the leaf veins to survive. Researchers have shown that on the rice leaf surface, macrogroves and millions of micro-papillas $(2-5 \mu \mathrm{m})$ covered with nanoscale surface features, namely macro-micro-nano threelevel structures, are the key to the anisotropic wetting phenomenon. ${ }^{9}$ To reproduce artificial surfaces with directional sliding wetting, a variety of methods, such as photolithography, ${ }^{8}$ reactive ion etching, ${ }^{12}$ and micro-molding, ${ }^{24}$ have been proposed to generate anisotropic microstructure grooves for mimicking the natural rice leaf. However, most of these anisotropic microstructures are parallel grooves without nanostructures and can only realize different contact angles along the parallel and perpendicular directions. In 2011, we demonstrated the anisotropic sliding angle (ASA) by designing threelevel macrogrooves (200-400 $\mu \mathrm{m})$ and micro/nanostructures. ${ }^{9}$ After this, other groups also realized the fabrication of macrogroove structures with a large period and height based on photolithography. ${ }^{\mathbf{1 0} 11}$ However, these methods require a complex multi-step process for two-level micro/nano structures and especially for three-level macro/micro/nano structures, e.g., square trenches fabricated by photolithography, PDMS transfer, and micro/nanostructure coating. Moreover, additional surface modification by chemical nanoparticles is 
required to form nanostructures on the substrates. Other microfabrication methods, including surface wrinkling by ultravioletozone radiation, ${ }^{13}$ soft transfer $^{15}$ and nanoimprint lithography, ${ }^{16}$ offer valuable platforms for investigating the anisotropic wetting property under the effect of surface nanostructures. Nevertheless, it is difficult to precisely control the period and height of the anisotropic grooves due to the limitations of the inherent fabrication method. Moreover, they only investigated the static anisotropic sliding property.

Laser machining has emerged as an innovative method to prepare special surface micro/nanostructures that overcomes the disadvantages of traditional methods. ${ }^{721-30}$ It is a one-step and simple method without a mask or harsh environment. In addition, the surface structures, even complex 3D structures, can be controlled by modulating the processing parameters. For instance, Chen et al. ${ }^{27}$ prepared line-patterned surfaces with controlled wetting ability based on femtosecond laser microfabrication on polydimethylsiloxane (PDMS) materials, and this process shows many advantages such as low cost, ease of use, good chemical inertness, etc. However, for a larger period groove, e.g., $200 \mu \mathrm{m}$, the contact angle (CA) decreased to $120^{\circ}$, and the water droplet does not roll down, even when the fabricated surface is turned upside down. This is because there are lots of flat untreated PDMS regions between the adjacent grooves. Therefore, it is of great interest to produce a leaf-like surface with anisotropic sliding on PDMS substrates and to study their dynamic process and potential applications.

For the first time, an energy-modulation femtosecond laser scanning strategy was proposed to realize three-level artificial rice leaf (macro and micro/nano) surfaces with periodic macrogrooves and hierarchical micronanostructures on PDMS film. The macrogrooves for anisotropic control were realized by larger-energy $\left(>0.40 \mathrm{~J} \mathrm{~cm}^{-2}\right)$ laser scanning, while the micro/ nanostructures for superhydrophobic ability were fabricated by small-energy $\left(0.08 \mathrm{~J} \mathrm{~cm}^{-2}\right)$ laser scanning. In order to quantitatively investigate the anisotropic wettability, the sliding angles of the fabricated surfaces with different periods and heights were systematically measured. We not only studied the static anisotropy and superhydrophobicity of the treated PDMS, but we also investigated the dynamic process of water droplet anisotropic sliding. The distinct ability of directional guide sliding and size-constrained fog deposition on anisotropic biosurfaces was demonstrated. The collection efficiency of water on an anisotropic surface with $5^{\circ}$ and $10^{\circ}$ rotation angles was four times and eighty times higher than that on an isotropic surface. These results show that the bionic rice leaf surface has an anisotropic ability comparable with natural ones.

\section{Experimental section}

\subsection{Materials}

In our experiment, the regular groove-array template was processed on a layer of PDMS precursor liquid mixed with the curing agent (10:1 by weight) and cured at $60^{\circ} \mathrm{C}$ for $3 \mathrm{~h}$. Here, PDMS Sylgard 184 purchased from Dow Corning (MI) was chosen for its good elasticity, high optical transmission, and biocompatibility. The thickness of the film was about $5 \mathrm{~mm}$.

\subsection{Structuring PDMS surface}

The $100 \mathrm{fs}$ pulses with a repetition rate of $1 \mathrm{kHz}$ at a central wavelength of $800 \mathrm{~nm}$ were generated from a regenerative amplified Ti:sapphire femtosecond laser system (Legend Elite$1 \mathrm{~K}-\mathrm{HE}$, Coherent, America), and the system was employed for irradiation. The laser beam was guided normally onto the PDMS surface by a galvo-scanner system (SCANLAB, Germany) through the free space. A $63 \mathrm{~mm}$ telecentric $\mathrm{f} \theta$ lens of the galvoscanner system provided a focused laser beam perpendicular to the sample. The diameter of the focal point was set at $250 \mu \mathrm{m}$ by adjusting the distance from the telecentric $\mathrm{f} \theta$ lens to the PDMS surface. The schematic fabrication process is shown in Fig. 1(d). First, the PDMS surface was irradiated with a scanning space of $50 \mu \mathrm{m}$ and pulse energy of $0.08 \mathrm{~J} \mathrm{~cm}^{-2}$ at a scanning speed of 1 $\mathrm{mm} \mathrm{s}^{-1}$. After laser ablation, the CAs of the rough surface were over $150^{\circ}$, while the SAs were $1.5 \pm 0.5^{\circ}$ in any direction. The anisotropic sliding angle of the parallel and perpendicular directions was about $0^{\circ}$, indicating a superhydrophobic surface with an isotropic wetting property formed. Then, to fabricate periodic groove structures, the pulse energy was set from 0.08 to $0.72 \mathrm{~J} \mathrm{~cm}^{-2}$, and the scanning space was changed from 100 to $600 \mu \mathrm{m}$ at the same scanning speed. The surfaces with period parallel groove structures showed the anisotropic wetting property as the SAs in parallel and perpendicular directions are different. Here, the surface with an isotropic wetting property is called an isotropic surface, while the surface with an anisotropic wetting property is called an anisotropic surface. At a scanning speed of $1 \mathrm{~mm} \mathrm{~s}^{-1}$, the total processing time was $15 \mathrm{~min}$ for a substrate with a length of $40 \mathrm{~mm}$ and width of $10 \mathrm{~mm}$ when the scanning space was $400 \mu \mathrm{m}$. With acetone, alcohol and deionized water, an ultrasonic bath was used to clean the asprepared samples for $10 \mathrm{~min}$ after laser irradiation.

\subsection{Characterization of real and bionic rice leaf surface}

The structural properties of the real and artificial rice leaf samples were characterized by a scanning electron microscope (SEM, JSM-6700F, JEOL, Tokyo, Japan). The solid surface composition was studied by X-ray photoelectron spectroscopy. The CAs and SAs of $5 \mu \mathrm{l}$ of water were measured by a contactangle system (CA100D, Innuo, China). Both the values of parallel and perpendicular CAs were averaged by measuring five drops at different locations on the same sample. To measure the parallel and perpendicular SAs, water drops (volume of $4 \mu \mathrm{l}$ ) were transferred to the level surfaces, and then the substrates were slowly tilted with an increment of $0.1^{\circ}$ until the droplets started to slide. Sequence images were taken every $100 \mathrm{~ms}$ by the contact-angle system equipped with a computer-controlled camera to show the sliding behavior of the water droplets on the inclined surfaces.

The directional sliding ability was investigated by the contact-angle system and a charge-coupled device (CCD) camera system to take photographs at 10 frames per second.

To study the fog collection of different surfaces, an ultrasonic atomizer was used to generate water droplets with a diameter of about $4 \mu \mathrm{m}$ [Fig. S1†]. The micrometer-sized droplet was transported by a curved $10 \mathrm{~cm}$ square-channel to 

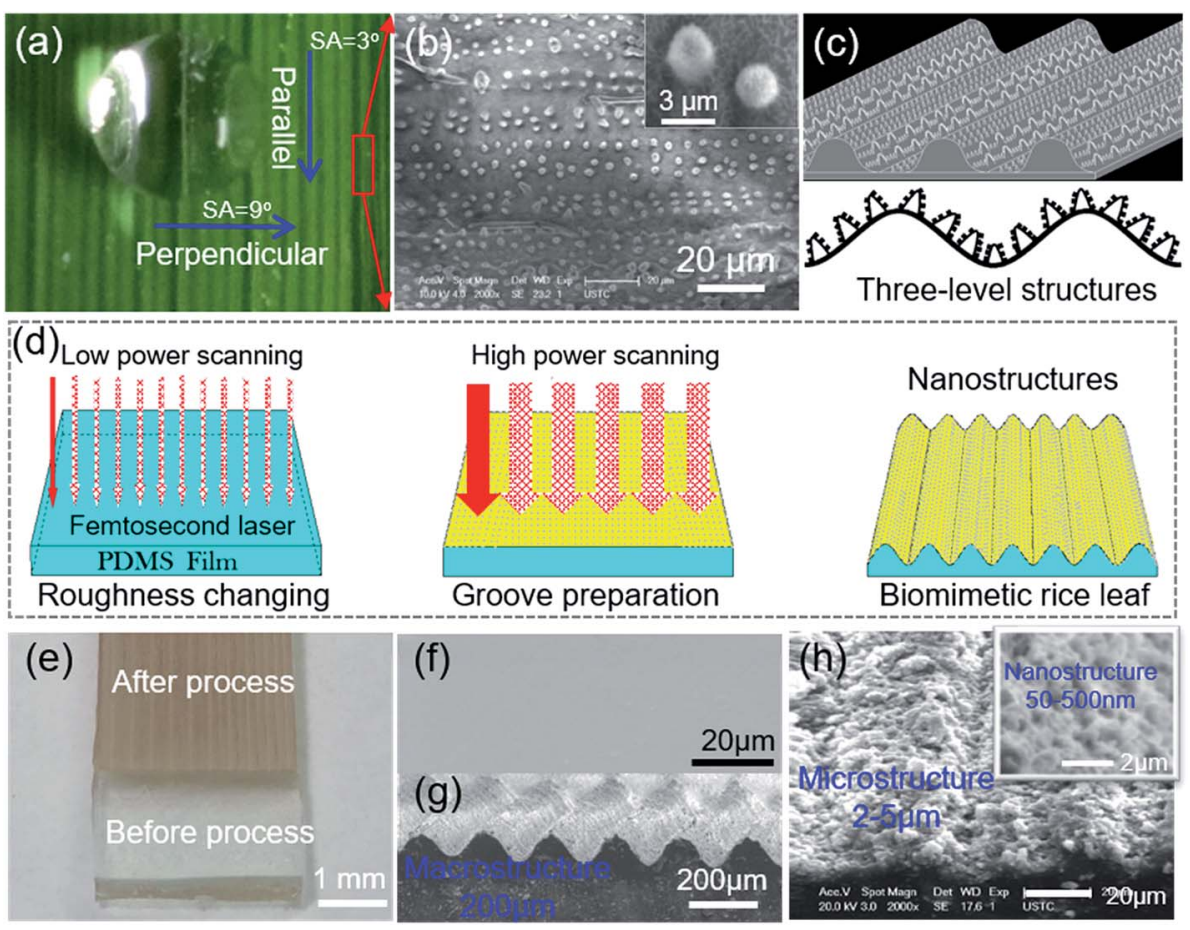

Fig. 1 Multi-level rice leaf surfaces with anisotropic sliding behavior and fabrication of the rice leaf-like biosurfaces. (a) The upside of rice leaf blades. (b) The top-view SEM image of the rice leaf upper surface. The inset is a magnified image of micropillars with nanostructures. (c) Hierarchical model of the rice leaf. (d) Schematic of the bionic rice leaf using a femtosecond laser. (e) Optical image of the processed PDMS film. (f) Top-view SEM of untreated PDMS. (g) Cross-sectional SEM image of the rice leaf-like wavy grooves. (h) Top-view SEM image of the processed PDMS surface, and the insert is a magnified image of micro-nanostructures.

the sample vertically. ${ }^{31,32}$ The humidity and temperature of the sample area were $68 \%$ and $25{ }^{\circ} \mathrm{C}$, respectively.

\section{Results and discussion}

\subsection{Multi-level structures of the rice leaf and the preparation} of the bionic rice leaf on PDMS

Shown in Fig. 1(a) is a piece of fresh green rice leaf blade, and the periodic parallel grooves are distributed on the surface. Topview SEM image in Fig. S2(a) and (b) $\dagger$ further shows that the groove period is about $200 \mu \mathrm{m}$. A droplet on the rice leaf blade displays superhydrophobic ${ }^{3}$ behavior with a water CA greater than $150^{\circ}$. Interestingly, the droplet rolls off more easily from the parallel direction (with a SA of $3^{\circ}$ ) than the perpendicular direction (with a SA of $9^{\circ}$ ) [Fig. S2(c) $\dagger$ ]. From the $80^{\circ}$ tilted SEM image [Fig. S2(d) $\dagger$ ], it is seen that the grooves consist of hierarchical micro/nanostructures. The further magnified SEM image [Fig. 1(b), S2(e) and (f) $\dagger$ ] indicates that the micro/ nanostructures are regularly distributed parallel to the grooves but irregularly distributed in a vertical direction. These mastoids about $2 \mu \mathrm{m}$ diameter are similar to that of other plants and insects with superhydrophobicity, such as the lotus leaf. ${ }^{33}$ The surface of the rice leaf consists of micrometer-scale papilla and nanostructures, which is called a two-level structure. However, with the groove structure, the rice leaf is a three-level macro/micro/nanostructure [Fig. 1(c)], thus, it exhibits a distinct anisotropic sliding property. ${ }^{3}$ In this physical model, periodic grooves with periods of $200 \mu \mathrm{m}$ were fabricated, which form an energy barrier for the droplet, determining the anisotropy ability of the rice leaf surface. Meanwhile, the papillary structures similar to the microstructures on the lotus leaf surface distribute on the grooves surfaces to form the Cassie-Baxter superhydrophobic state. ${ }^{1}$ The combined effect of the grooves and papillas endow the rice leaf with anisotropic superhydrophobicity. ${ }^{9}$

Then, an artificial rice leaf was prepared by one-step energymodulation femtosecond laser microfabrication [Fig. 1(d)] on a flat PDMS surface [Fig. 1(f)] at the same scanning speed but different scanning space and pulse energy. The solid arrows in Fig. 1(d) represent the first scanning rows, and the dotted arrows are the second scanning rows. First, the roughness of the surface of the PDMS film was formed by femtosecond laser irradiation with a scanning space of $50 \mu \mathrm{m}$ at a low power of $0.08 \mathrm{~J} \mathrm{~cm}^{-2}$, which contributed to the superhydrophobicity and low adhesion force, just like the lotus leaf surface. Then, femtosecond laser scanning with a higher pulse energy $(0.40 \mathrm{~J}$ $\left.\mathrm{cm}^{-2}\right)$ and larger scanning space $(200 \mu \mathrm{m})$ was conducted to produce the macrogrooves. By this scanning strategy, a threelevel artificial rice leaf was realized, as shown in Fig. 1(h). When the scanning space and pulse energy were set to $200 \mu \mathrm{m}$ and $0.24 \mathrm{~J} \mathrm{~cm}^{-2}$, wavy grooves with a period of $200 \mu \mathrm{m}$ and height of $50 \mu \mathrm{m}$, just like the rice leaf, were also formed [Fig. 1(g)]. The magnified SEM image of Fig. 1(h) shows that the macrogrooves were covered by micro/nanostructures. 


\subsection{Quantitative investigation of the relationship between} anisotropic sliding and groove parameters

In order to systemically investigate the relationship between the wetting and the grooves parameters, samples with a groove period ranging from 100 to $600 \mu \mathrm{m}$ and a depth ranging from 30 to $100 \mu \mathrm{m}$ [Fig. 2(a)-(f)] were prepared by simply controlling the processing parameters, namely the scanning space and pulse energy. As the pulse energy increased from $0.08 \mathrm{~J} \mathrm{~cm}^{-2}$ to $0.72 \mathrm{~J}$ $\mathrm{cm}^{-2}$, the depth of the groove became larger. Fig. 2(b)-(e) show that the processing depth of the microgrooves increased from 30 to $90 \mu \mathrm{m}$ with the laser pulse energy increasing from 0.08 to $0.56 \mathrm{~J} \mathrm{~cm}^{-2}$. However, it was noticed that when the pulse energy was raised to $0.72 \mathrm{~J} \mathrm{~cm}^{-2}$ [Fig. 2(f)], the strong pulse energy overlapped between the laser spots of the adjacent grooves and weakened the increase in the groove depth, which resulted in the formation of sharper grooves.

To investigate the anisotropic wettability of the bionic rice leaf, static water CAs [Fig. 2(g)] and dynamic water SAs were measured in both the perpendicular and parallel directions. As shown in Fig. 2(g), the CAs in both directions were larger than $150^{\circ}$, which indicated that all the processed PDMS surfaces became superhydrophobic. The solid surface chemical composition was measured by X-ray photoelectron spectroscopy (XPS) [Fig. S3 and Table S1†]. There is slight change (about $8 \%$ ) on the
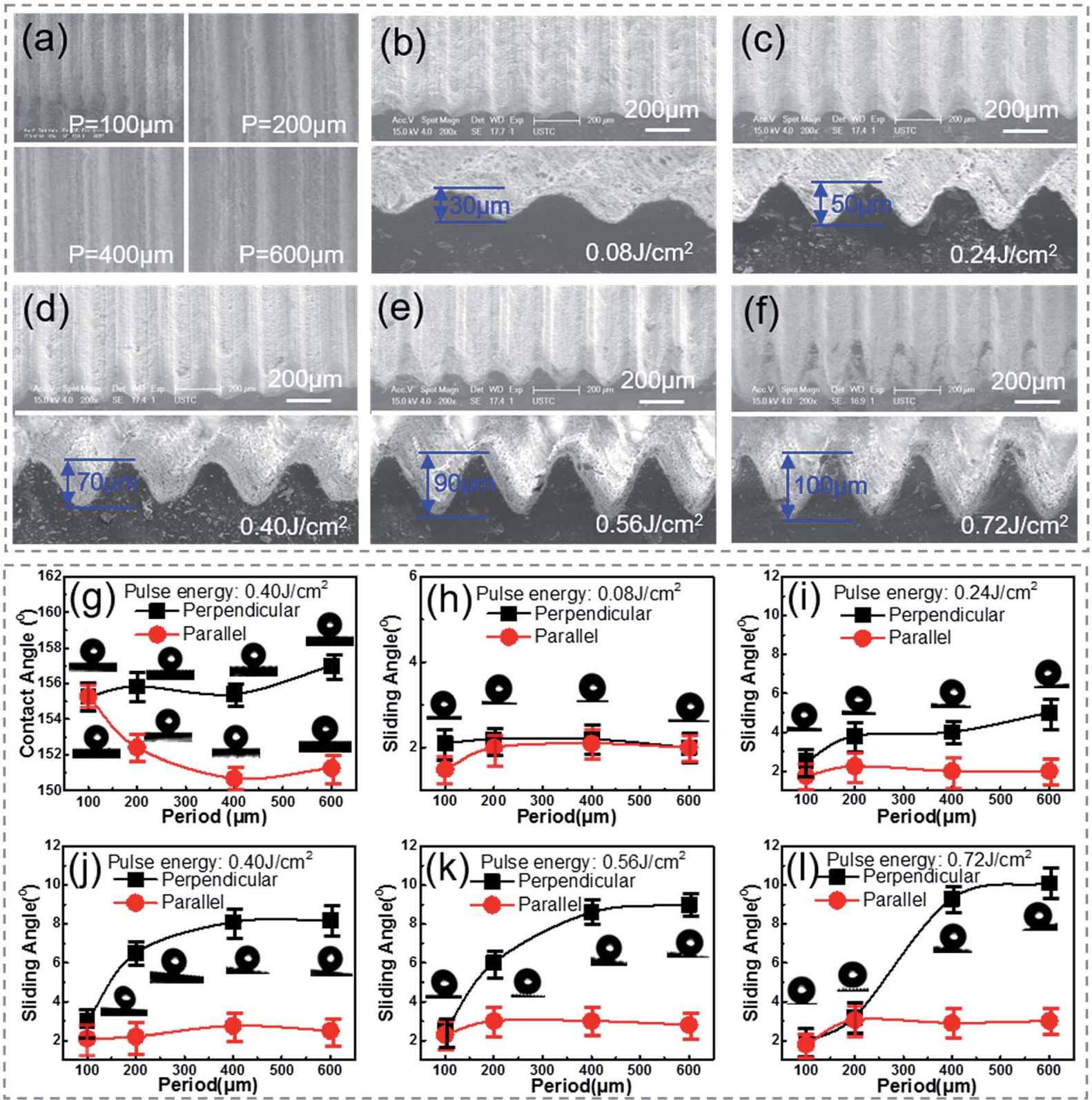

Fig. 2 Bionic rice leaf with different morphologies and surface wettability measurements (a) bionic rice leaf with periods 100, 200, 400, and 600 $\mu \mathrm{m}$. (b) - (f) $200 \mu \mathrm{m}$ microgrooves with depths of 30,50, 70, 90, and $100 \mu \mathrm{m}$. (g) Perpendicular and parallel water contact angles of the bionic surface with a pulse energy of $0.40 \mathrm{~J} \mathrm{~cm}^{-2}$ and period from 100 to $600 \mu \mathrm{m}$. (h) -(l) Dynamic water sliding angles (SAs) in both the perpendicular and parallel direction of grooves with the contrast of different width and height. 
XPS signal C and O, which corresponds to previous research. ${ }^{34-37}$ The reason may be that carbon oxides formed during the fs laser irradiation. This does not change the surface energy significantly because the CA increased from $110^{\circ}$ (flat surface) to $160^{\circ}$ (microstructured surface). ${ }^{38}$ The measured SAs [Fig. 2(h)-(l)] show that the parallel SAs along the direction of the macrogrooves are very small, about $2-3^{\circ}$ for all bionic rice leaf surfaces. However, samples with a higher pulse energy $(>0.24 \mathrm{~J}$ $\left.\mathrm{cm}^{-2}\right)$ and larger period $(>200 \mu \mathrm{m})$ have greater SAs in the perpendicular direction [ESI $\dagger$ Video 1], similar to the natural rice leaf. For the sample with a pulse energy of $0.40 \mathrm{~J} \mathrm{~cm}^{-2}$ and period of $400 \mu \mathrm{m}$, the measured anisotropy was $6^{\circ}$ with SAs in the perpendicular and parallel direction of about $9^{\circ}$ and $3^{\circ}$, respectively. So, the variation in the contact and rolling angles with the period of the grooves structure [Fig. 2] is small because of their excellent superhydrophobic ability. The variation of the rolling angle with the period of the grooves structure is about $1^{\circ}$, $3^{\circ}, 6^{\circ}, 6^{\circ}, 7^{\circ}$ [Fig. 2(g)-(l)] for $30,50,70,90,100 \mu \mathrm{m}$ grooves, respectively. This experimental result agreed with that of the natural rice leaf $\left(6^{\circ}\right)$. Thus, our femtosecond laser induced biomimetic surfaces are a convenient and efficient method to mimic the anisotropic sliding property of rice leaves. Here, the groove heights were calculated according to the ratio of the groove depth to the period in the SEM images by measuring the distance between the groove bottom to the top [Fig. S4(a)-(d)†]. The obvious air gaps under the droplets in the optical microscope images of Fig. S4(e)-(g) $\dagger$ show that the sample was always in the Cassie-Baxter state when the depth and period were changed. Here, from the height of the air gaps in the images, we inferred the barrier height disparity of the different surface macrogrooves. The relationship between the microgroove profile and three-phase contact mode is schematically illustrated in Fig. 3 to explain the potential mechanism of the surface anisotropic sliding property.

Grooves with narrow widths $(<100 \mu \mathrm{m})$ have no obvious anisotropic sliding property. In our experiment, the width depends on both the scanning space and pulse energy. When the scanning space is less than $100 \mu \mathrm{m}$ [Fig. 3], the anisotropy (about $1^{\circ}$ ) has no difference, even when the pulse energy is increased from 0.08 to $0.72 \mathrm{~J} \mathrm{~cm}^{-2}$ [Fig. 2(h)-(l)]. In Fig. 2(c), the anisotropy for the $200 \mu \mathrm{m}$ width groove with a smaller height $(<50 \mu \mathrm{m})$ is still not clear [Fig. 2(i)], while the PDMS surfaces with larger widths $(>200 \mu \mathrm{m})$ and depths $(>70 \mu \mathrm{m})$ show an apparent anisotropic sliding property of $5-6^{\circ}$ [Fig. $2(\mathrm{j})$ and $(\mathrm{k})$ ]. The reason may be that droplet on the narrower grooves with a smaller depth has a smaller barrier height $(\Delta h)$ inside the surface structure and results in weak anisotropy. The energy barrier provided by the grooves with a smaller depth [Fig. 2(b)] was not able to limit droplet movement along the perpendicular direction compared to the grooves with a larger depth. With the increase in the groove width and depth, the barrier height, $\Delta h$, becomes larger [Fig. 3], leading to strong anisotropy. ${ }^{9}$ Furthermore, it can be seen in Fig. 2(l) that the anisotropic SA of the surfaces from the higher pulse energy and larger scanning space corresponding to a larger width and deeper depth is even higher than $7^{\circ}$. According to the measurement and analysis, the arrays of the larger microgrooves are the decisive factor for anisotropic sliding, while the grooves with a small width and depth do not have the ability for directional sliding. By comparing the ASA of surfaces produced by the same scanning space $(200 \mu \mathrm{m})$ but different pulse energy $(0.40$, 0.56 , and $0.72 \mathrm{~J} \mathrm{~cm}^{-2}$ ) [Fig. 2(j)-(l)], it was seen that the difference between the parallel and perpendicular sliding angles decreases. This is because the surface roughness will increase as a result of the high energy overlapping effect $\left(0.72 \mathrm{~J} \mathrm{~cm}^{-2}\right)$ while the

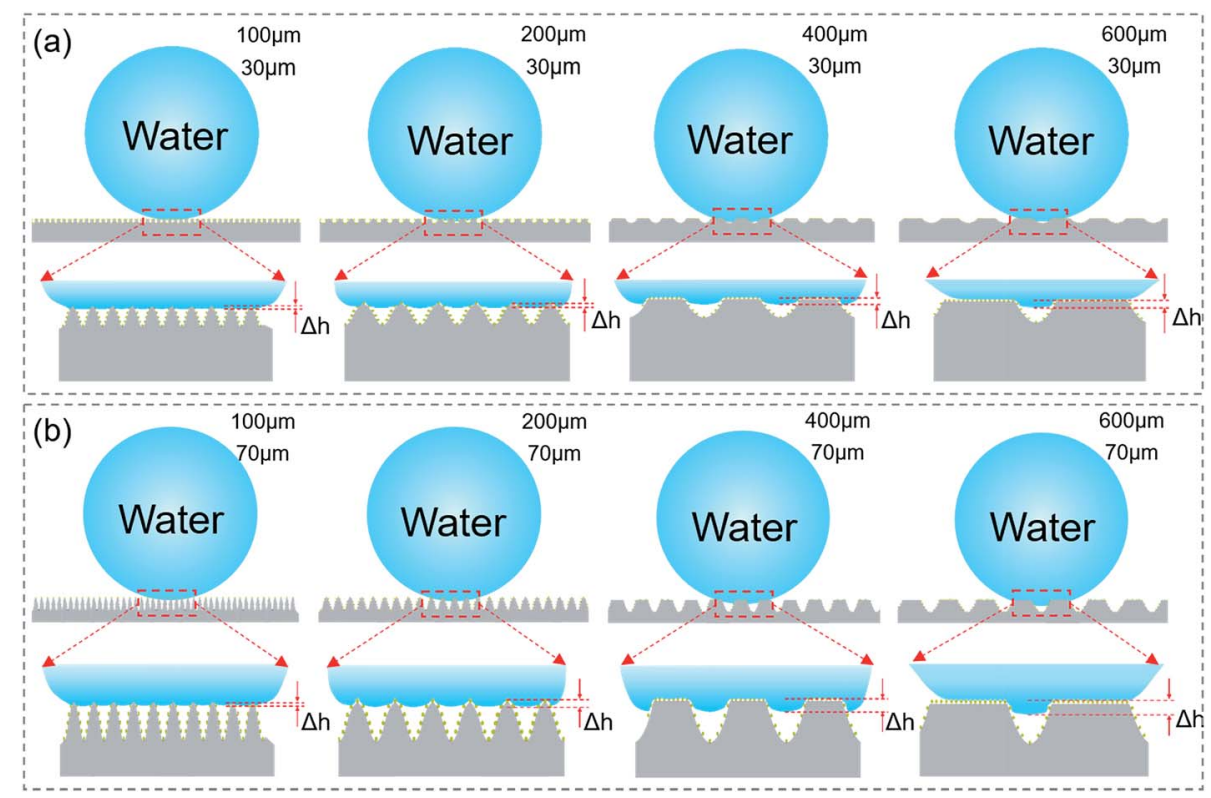

Fig. 3 Schematic of the water droplet contact mode with the laser-induced microgrooves and the macrogroove morphology. (a) The first line shows surface grooves with a depth of $30 \mu \mathrm{m}$ while the period varies from 100 to $600 \mu \mathrm{m}$. The second line is the detailed contact mode for substrates with different periods from the first line. (b) The first line shows surface grooves with a depth of $70 \mu \mathrm{m}$ while the period varies from 100 to $600 \mu \mathrm{m}$. The second line is the detailed contact mode for substrates with different periods from the first line. 
scanning space is relatively small $(200 \mu \mathrm{m})$. The CAs in both the parallel and perpendicular direction for the $200 \mu \mathrm{m}$ width and $100 \mu \mathrm{m}$ height grooves were larger than $160^{\circ}$, which means they have a high roughness [Fig. S5(c)†]. In addition, we also immersed the sample in water to measure the contact angle deterioration [Fig. S6†]. The contact angle decreased from about $155.4 \pm 1^{\circ}$ to $146.3 \pm 1^{\circ}$ when the sample was immersed in water for 3 days. The CA recovered to $152.2 \pm 2^{\circ}$ after the substrate was exposed to air for 3 days. This phenomenon indicates that the micro-nano structures on the substrate can be used many times. Furthermore, one sample exposed to air for 12 months was still superhydrophobic, as shown in Fig. S5(d), $\dagger$ which means the bionic surface is stable. We suppose that the micro/ nanostructures not only made the surface superhydrophobic but also protected the surface from significant contact angle deterioration. ${ }^{39}$ (a)

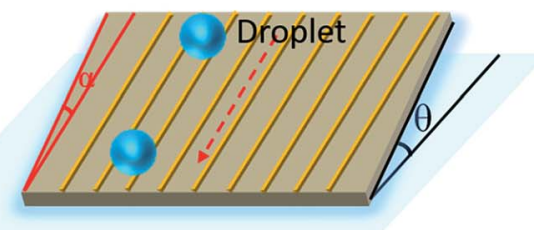

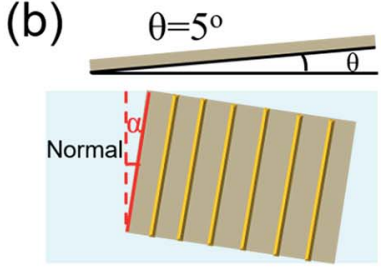

(c)

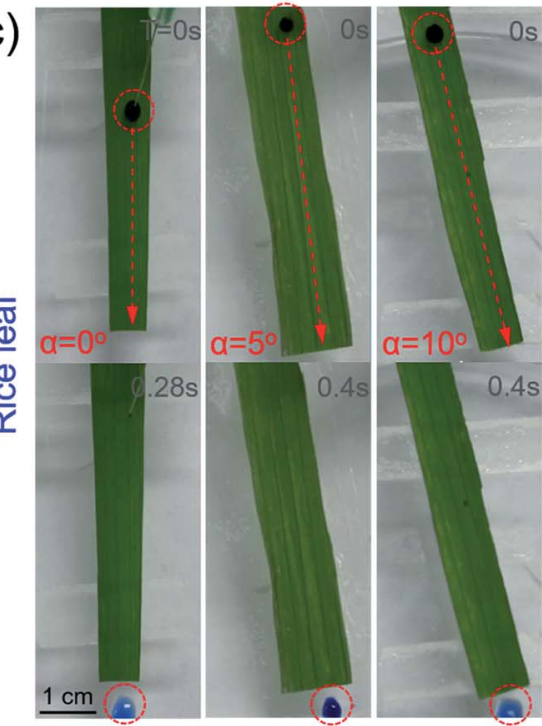

(e)

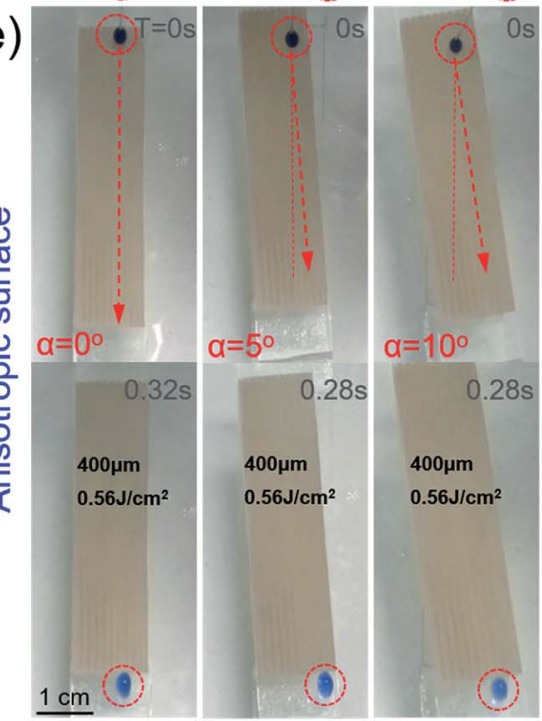

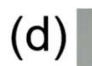
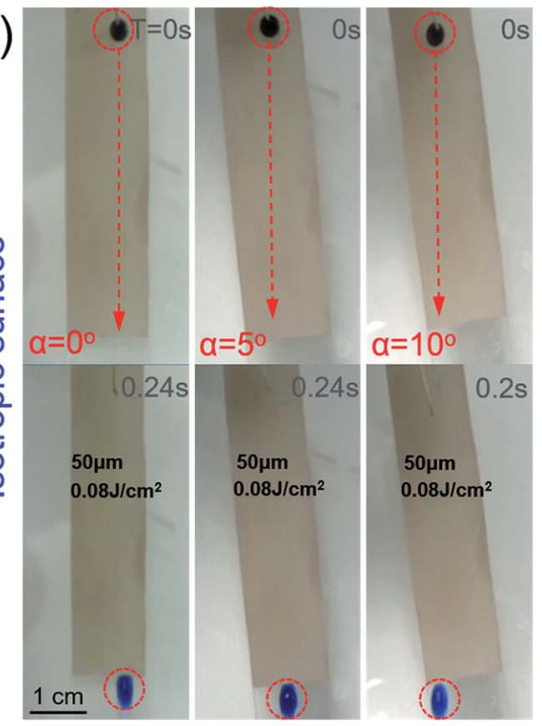

(f)

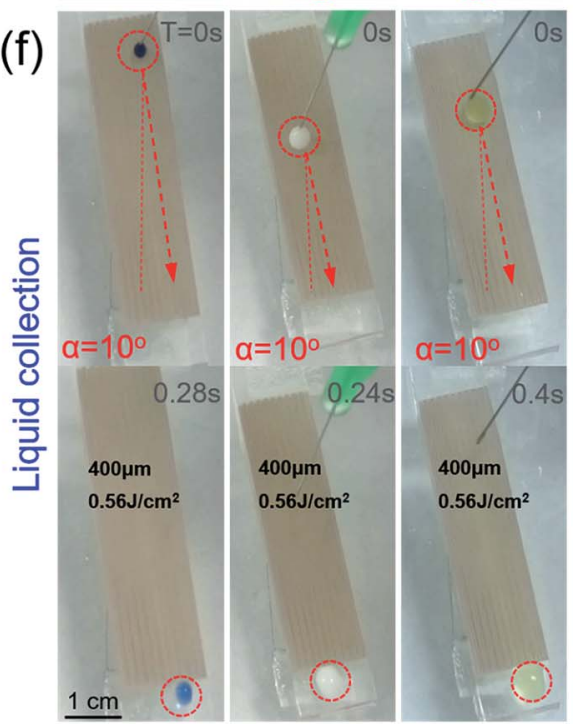

Fig. 4 Comparison of dynamic anisotropic sliding behaviors on rice leaf, isotropic and anisotropic surfaces. (a) Water droplet on the inclined surface with a rotation angle $\alpha$, which is vertical to the grooves, results in directional sliding along the parallel direction of the grooves. (b) The inclined angle $\theta$ of the substrates is $5^{\circ}$ while the rotation angle ranges from $0^{\circ}$ to $10^{\circ}$. (c)-(e) Ink droplet rolling off the rice leaf, isotropic surface and anisotropic surface with rotation angles from 0 to $10^{\circ}$, respectively. (f) Ink, milk and juice rolling off the bionic rice leaf surface along the groove arrays with the sample body tilted at $10^{\circ}$. 


\subsection{Dynamic anisotropic sliding and directional water collection}

Water droplets can easily roll along the parallel direction of the rice leaf to the root, keeping the plant nourished by water. To investigate the effect of three level macrogrooves, ${ }^{\mathbf{4 0 - 4 2}}$ we further compared the Directional Guide Sliding (DGS) of the isotropic surface and anisotropic surface. As shown in the schematic diagram of DGS [Fig. 4(a) and (b)], the sample was placed on a substrate with an inclined angle $\theta$, which is the angle between the sample and the horizontal plane. The rotation angle $\alpha$ is the angle between the macrogrooves and the normal. The inclined angle $\theta$ was set to be $5^{\circ}$ to provide potential energy, while the rotation angle $\alpha$ ranged from $0^{\circ}$ to $10^{\circ}$. The droplets could easily roll down, and the sliding direction was indicated by the red arrows in Fig. 4(c)-(e). In Fig. 4(c), the blue ink droplet rolled off the natural rice leaf surface along the groove arrays rapidly without dispersion, even when the rice leaf was rotated 10 degrees. It can be seen that the droplets on isotropic surfaces processed with a scanning space of $50 \mu \mathrm{m}$ and pulse energy of $0.08 \mathrm{~J} \mathrm{~cm}^{-2}$ [Fig. 4(d)] rolled down vertically by the effect of gravity only. However, anisotropic surfaces fabricated with a period of $400 \mu \mathrm{m}$ and pulse energy of $0.56 \mathrm{~J} \mathrm{~cm}^{-2}$ can guide droplets to roll along grooves with an $\alpha$ changed from $0^{\circ}$ to $10^{\circ}$ [Fig. 4(e)], displaying similar dynamic anisotropic wetting ability to that of the natural rice leaf [ESI $\dagger$ Video 2]. These results further verify that macrogroove arrays with micro/nano hierarchical structures on the rice leaf lead to anisotropic sliding. The schematic force analysis of the water droplet on the inclined bionic rice leaf is shown in Fig. S7 in the ESI. $\uparrow$ Furthermore, the bionic rice leaf exhibited a similar DGS property for different kinds of liquid, regardless of the adhesion force difference [Fig. 4(f)]. It is considered that the DGS property plays a critical role in the liquid collection function, and it can be widely used in various liquid transports in the biomedical field.

To quantitatively examine the liquid anisotropic sliding function of the artificial bionic surface, a liquid collection experiment was conducted [Fig. 5]. Water $(10 \mathrm{ml})$ was added to the arbitrary points of the irradiated area and was collected by a container [ESI $\dagger$ Video 3]. Here, a parameter called the Collection Efficiency (CE) was introduced to evaluate the liquid anisotropic sliding function. The $\mathrm{CE}$ is defined as the ratio of the collected water and total water that was dropped onto the sample. The formula is as follows:

$$
\text { Collection efficiency }=V_{\text {collected }} / V
$$

where $V_{\text {collected }}$ and $V$ are the volume of the water collected by the container and the total water, respectively.

\section{(a) Isotropic surface}
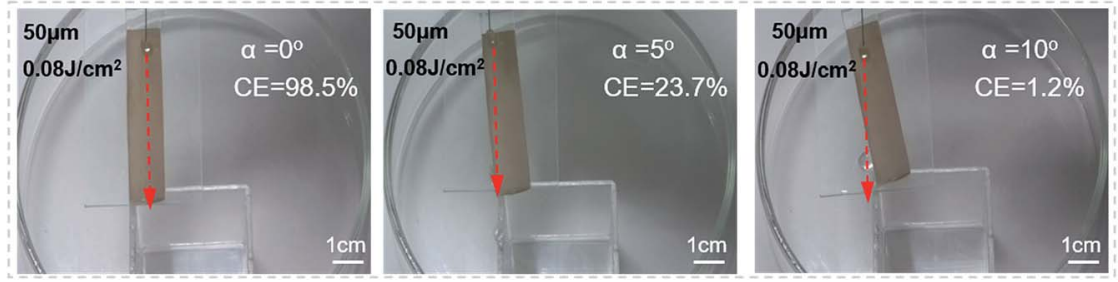

(b)
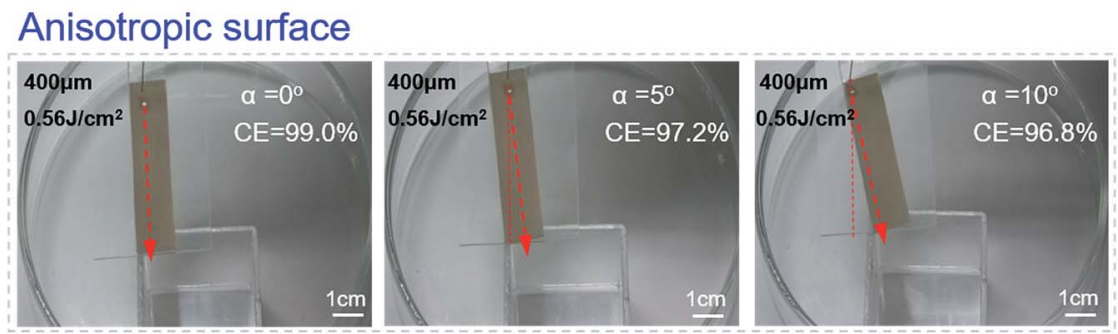

(c)

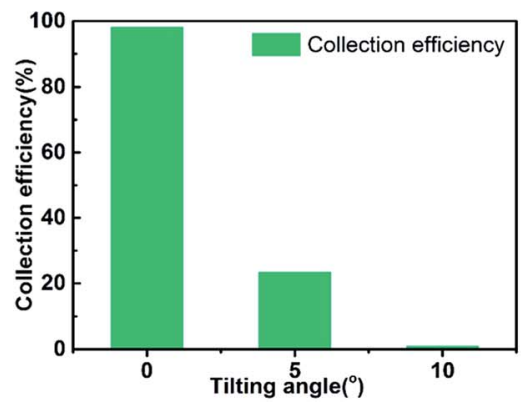

(d)

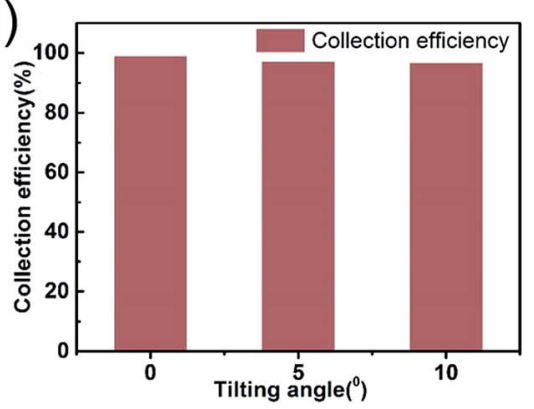

Fig. 5 Comparison of the liquid collection efficiency on isotropic and anisotropic surfaces under different rotation angles. (a) and (b) Water collection with rotation angles of $0,5,10^{\circ}$ on isotropic surface and anisotropic surface. (c) and (d) Water collection efficiency histogram of the isotropic surface and anisotropic surface. 

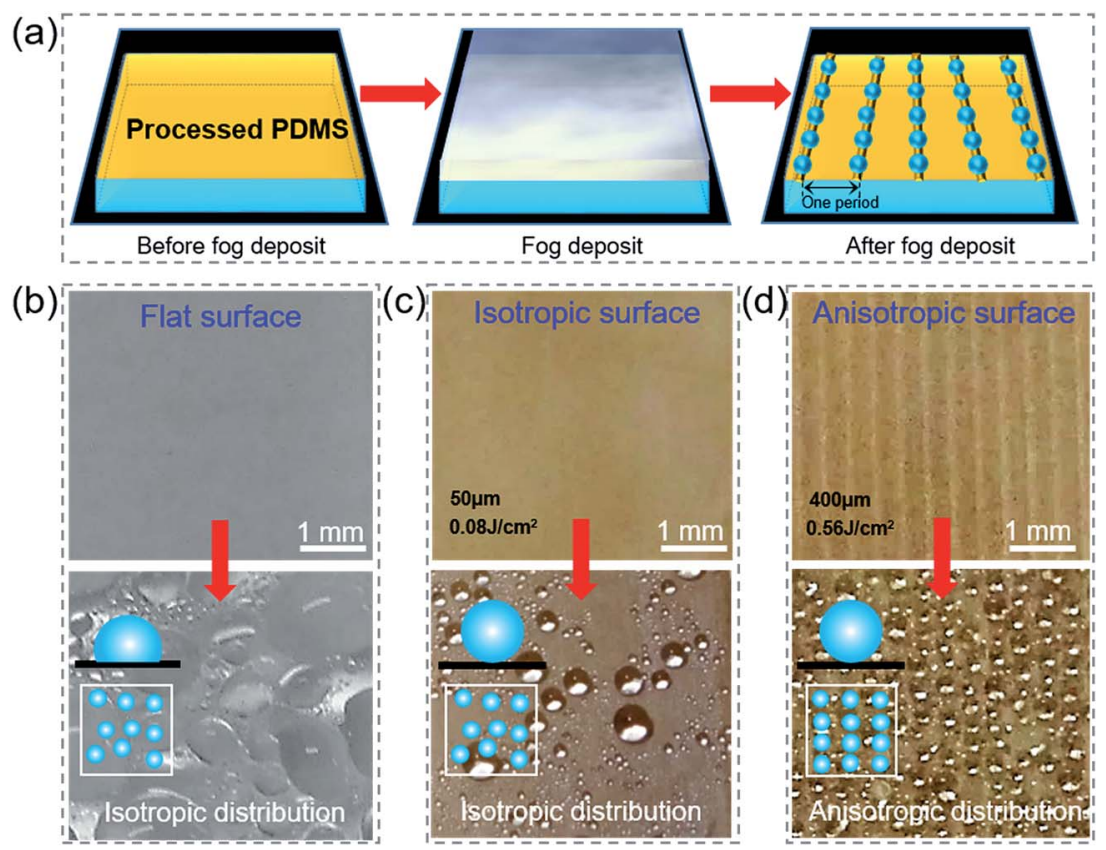

Fig. 6 Directional and size-constrained fog deposition. (a) Schematic of the fog deposition process. (b)-(d) Images of flat, isotropic and anisotropic surfaces before and after fog deposition. The inset schematic images indicate the contact and distribute mode of each surface.

The PDMS on the slide was rotated with different angles of $0^{\circ}, 5^{\circ}$, and $10^{\circ}$ [Fig. 5(a) and (b)]. With the rotation angle increasing, the CE of the isotropic PDMS surface decreased from $98.5 \%$ to $1.2 \%$ [Fig. 5(a) and (c)]. However, the anisotropic surface showed great directional guidance of the droplet (anisotropic) sliding ability with few decreases $(0.8 \%)$ of $\mathrm{CE}$ [Fig. 5(b) and (d)]. These further demonstrated the strong water droplet control ability of the anisotropic surface due to the special three-level macro/micro/nanostructures.

\subsection{Directional and size-constrained fog deposition}

Water vapor liquefaction on the leaf, as an important ecological phenomenon in the paddy field, ${ }^{43}$ has multiple effects on the growth of rice. It can be easily absorbed by plants at night to keep the canopy moisture, reduce crop transpiration, and also supply necessary nutrients like nitrogen and phosphorus. ${ }^{44}$

To investigate the influence of periodic surface trench structures on the liquid settlement and compare the fog collection ability on flat, isotropic and anisotropic surfaces, the deposition of micrometer-sized water droplets, called fog, was demonstrated in our experiment. As shown in Fig. 6(a), the initial substrate was dry, and it was placed under a wet air flow for $30 \mathrm{~s}$. From the images of the different surfaces before and after the fog deposit [Fig. 6(b)-(d)], it can be seen that the water on the flat PDMS film showed various sizes (from 20 to $700 \mu \mathrm{m}$ ), while the one on the anisotropic film showed uniform sizes (from 200 to $400 \mu \mathrm{m}$ ). Moreover, the liquid beads on the anisotropic surface were arranged along the trench direction due to the bionic rice leaf. The macrogrooves regulated drop growth and inhibited fog coalescence so that the fog was distributed along the parallel direction. This property helps a rice leaf collect dew and flow water to its root, which is crucial for their living in nature.

\section{Conclusion}

In summary, inspired by the rice leaf surfaces, the hierarchical period macrogrooves covered with micro/nanostructures were prepared through one-step energy-modulation femtosecond laser irradiation on PDMS film surfaces. By tuning the scanning space and pulse energy, the profile of the macrogrooves can be effectively controlled. The effects of the hierarchical macrogroove surface morphology on the anisotropic sliding ability (from $0.2^{\circ}$ to $7.1^{\circ}$ ) and water collection (from $96.8 \%$ to $99.0 \%$ ) were systematically studied. In addition, the anisotropic wettability of the fog collection was also demonstrated. The fabricated PDMS surface was superhydrophobic and had anisotropic wetting comparable to the rice leaf. These results revealed the principle for the anisotropic wetting properties of the rice leaf and also provide a simple but efficient method for the design of functional surfaces in micro-fluidics devices, chemical and biological sensing, biomedicine, fuel cells, catalysts and other bioinspired system applications.

\section{Acknowledgements}

This work is supported by National Science Foundation of China (No. 51275502, 61475149, 51405464, 91223203, 61505047, and 11204250), Anhui Provincial Natural Science Foundation (No. 1408085ME104), National Basic Research Program of China (No. 2011CB302100), National Key Scientific Apparatus Development Project of China (No. 2013YQ220893), the Fundamental Research Funds for the Central Universities (No. WK2090000006 and 
WK2090090018), 111 project and "Chinese Thousand Young Talents Program".

\section{References}

1 Y. Zheng, X. Gao and L. Jiang, Directional Adhesion of Superhydrophobic Butterfly Wings, Soft Matter, 2007, 3, 178-182.

2 E. Bormashenko, Y. Bormashenko, T. Stein, G. Whyman and E. Bormashenko, Why do Pigeon Feathers Repel Water? Hydrophobicity of Pennae, Cassie-Baxter Wetting Hypothesis and Cassie-Wenzel Capillarity-induced Wetting Transition, J. Colloid Interface Sci., 2007, 311, 212-216.

3 L. Feng, S. Li, Y. Li, H. Li, L. Zhang, J. Zhai, Y. Song, B. Liu, L. Jiang and D. Zhu, Super-Hydrophobic Surfaces: From Natural to Artificial, Adv. Mater., 2002, 14, 1857-1860.

4 Y. Zheng, H. Bai, Z. Huang, X. Tian, F. Nie, Y. Zhao, J. Zhai and L. Jiang, Directional Water Collection on Wetted Spider Silk, Nature, 2010, 463, 640-643.

5 J. Ju, H. Bai, Y. Zheng, T. Zhao, R. C. Fang and L. Jiang, A Multi-structural and Multi-functional Integrated Fog System in Cactus, Nat. Commun., 2012, 3, 1247.

6 K. Li, J. Ju, Z. X. Xue, J. Ma, L. Feng, S. Gao and L. Jiang, Structured Cone Arrays for Continuous and Effective Collection of Micron-sized Oil Droplets from Water, Nat. Commun., 2013, 4, 2276.

7 J. Yong, F. Chen, Q. Yang, G. Du, C. Shan, H. Bian, U. Farooq and $\mathrm{X}$. Hou, Bioinspired Transparent Underwater Superoleophobic and Anti-oil Surfaces, J. Mater. Chem. A, 2015, 3, 9379-9384.

8 T. Kim and K. Suh, Unidirectional Wetting and Spreading on Stooped Polymer Nanohairs, Soft Matter, 2009, 5, 4131-4135.

9 D. Wu, J. Wang, S. Wu, Q. Chen, S. Zhao, H. Zhang, H. Sun and L. Jiang, Three-Level Biomimetic Rice-Leaf Surfaces with Controllable Anisotropic Sliding, Adv. Funct. Mater., 2011, 21, 2927-2932.

10 X. Yang, J. Song, W. Xu, X. Liu, Y. Lu and Y. Wang, Anisotropic sliding of multiple-level biomimetic rice-leaf surfaces on aluminium substrates, Micro Nano Lett., 2013, 8, 801-804.

11 G. D. Bixler, A. Theiss, B. Bhushan and S. C. Lee, Anti-fouling properties of microstructured surfaces bio-inspired by rice leaves and butterfly wings, J. Colloid Interface Sci., 2014, 419, 114-133.

12 D. Zhu, X. Li, G. Zhang, X. Zhang, X. Zhang, T. Wang and B. Yang, Mimicking the Rice Leaf-From Ordered Binary Structures to Anisotropic Wettability, Langmuir, 2010, 26, 14276-14283.

13 S. Lee, H. Lim, D. Lee, D. Kwak and K. Cho, Tunable Anisotropic Wettability of Rice Leaf-Like Wavy Surfaces, Adv. Funct. Mater., 2013, 23, 547-553.

14 S. Kang, C. Lee, H. Kim, B. Lee, J. Lee, M. Kwak and K. Suh, Directional Oil Sliding Surfaces with Hierarchical Anisotropic Groove Microstructures, Adv. Mater., 2013, 25, 5756-5761.

15 J. Yao, J. Wang, Y. Yu, H. Yang and Y. Xu, Biomimetic fabrication and characterization of an artificial rice leaf surface with anisotropic wetting, Chin. Sci. Bull., 2012, 57, 2631-2634.

16 B. Radha, S. H. Lim, M. Saifullah and G. U. Kulkarni, Metal hierarchical patterning by direct nanoimprint lithography, Sci. Rep., 2012, 3, 1078.

17 P. Zhang, H. Liu, J. Meng, G. Yang, X. Liu, S. Wang and L. Jiang, Grooved Organogel Surfaces towards Anisotropic Sliding of Water Droplets, Adv. Mater., 2014, 26, 3131-3135.

18 N. Malvadkar, M. Hancock, K. Sekeroglu, W. Dressick and M. Demirel, An Engineered Anisotropic Nanofilm with Unidirectional Wetting Properties, Nat. Mater., 2010, 9, 1023-1028.

19 S. Wu, D. Wu, J. Yao, Q. Chen, J. Wang, L. Niu, H. Fang and H. Sun, One-Step Preparation of Regular Micropearl Arrays for Two-Direction Controllable Anisotropic Wetting, Langmuir, 2010, 26, 12012-12016.

20 D. Wu, Q. Chen, J. Yao, Y. Guan, J. Wang, L. Niu, H. Fang and H. Sun, A Simple Strategy to Realize Biomimetic Surfaces with Controlled Anisotropic Wetting, Appl. Phys. Lett., 2010, 96, 053704.

21 S. Wu, J. Wang, L. Niu, J. Yao, D. Wu and A. Li, Reversible Switching between Isotropic and Anisotropic Wetting by Onedirection Curvature Tuning on Flexible Superhydrophobic Surfaces, Appl. Phys. Lett., 2011, 98, 081902.

22 D. Xia, L. Johnson and G. Lopez, Anisotropic Wetting Surfaces with One-Dimensional and Directional Structures: Fabrication Approaches, Wetting Properties and Potential Applications, Adv. Mater., 2012, 2, 1287-1302.

23 S. Wang, T. Wang, P. Ge, P. Xue, S. Ye, H. Chen, Z. Li, J. Zhang and B. Yang, Controlling Flow Behavior of Water in Microfluidics with a Chemically Patterned Anisotropic Wetting Surface, Langmuir, 2015, 31, 4032-4039.

24 J. Gao, Y. Liu, H. Xu, Z. Q. Wang and X. Zhang, Mimicking Biological Structured Surfaces by Phase-Separation Micromolding, Langmuir, 2009, 25, 4365-4369.

25 F. Chen, D. Zhang, Q. Yang, X. Wang, B. Dai, X. Li, X. Hao, Y. Ding, J. Si and X. Hou, Anisotropic Wetting on Microstrips Surface Fabricated by Femtosecond Laser, Langmuir, 2010, 27, 359-365.

26 G. Li, Y. Lu, P. Wu, Z. Zhang, J. Li, W. Zhu, Y. Hu, D. Wu and J. Chu, Fish Scales Inspired Design of Underwater Superoleophobic Microcones Arrays by Sucrose Solution Assisted Femtosecond Laser Irradiation for Multifunctional Liquid Manipulation, J. Mater. Chem. A, 2015, 3, 18675.

27 J. Yong, Q. Yang, F. Chen, D. Zhang, U. Farooq, G. Du and X. Hou, A Simple Way to Achieve Superhydrophobicity, Controllable Water Adhesion, Anisotropic Sliding, and Anisotropic Wetting Based on Femtosecond Laser-induced Line-patterned Surfaces, J. Mater. Chem. A, 2014, 2, 5499-5507. 28 G. Li, J. Li, C. Zhang, Y. Hu, X. Li, J. Chu, W. Huang and D. Wu, Large-area One-step Assembly of Threedimensional Porous Metal Micro/nanocages by Ethanolassisted Femtosecond Laser Irradiation for Enhanced Antireflection and Hydrophobicity, ACS Appl. Mater. Interfaces, 2014, 7, 383-390.

29 J. Yong, Q. Yang, F. Chen, D. Zhang, G. Du, H. Bian, J. Si, F. Yun and X. Hou, Superhydrophobic PDMS Surfaces with 
Three-dimensional (3D) Pattern-dependent Controllable Adhesion, Appl. Surf. Sci., 2014, 288, 579-583.

30 J. L. Yong, F. Chen, Q. Yang and X. Hou, Femtosecond Laser Controlled wettability of solid surfaces, Soft Matter, 2015, 11, 8897-8906.

31 T. Yu, J. Park, H. Lim and K. Breuer, Fog Deposition and Accumulation on Smooth and Textured Hydrophobic Surfaces, Langmuir, 2012, 28, 12771-12778.

32 S. Yang, J. Ju, Y. C. Qiu, Y. He, X. Wang, S. Dou, K. Liu and L. Jiang, Peanut Leaf Inspired Multifunctional Surfaces, Small, 2014, 10, 294-299.

33 L. Jiang, Y. Zhao and J. Zhai, A Lotus-Leaf-like Superhydrophobic Surface: A Porous Microsphere/Nanofiber Composite Film Prepared by Electrohydrodynamics, Angew. Chem., Int. Ed., 2004, 116, 4438-4441.

34 B. Schnyder, T. Lippert, R. Kötz, A. Wokaun, V. M. Graubner and O. Nuyken, UV-irradiation induced modification of PDMS films investigated by XPS and spectroscopic ellipsometry, Surf. Sci., 2003, 532, 1067-1071.

35 R. Srinivasan and S. Lazare, Modification of polymer surfaces by far-ultraviolet radiation of low and high (laser) intensities, Polymer, 1985, 26, 1297-1300.

36 S. Lazare, P. D. Hoh, J. M. Baker and R. Srinivasan, Controlled modification of organic polymer surfaces by continuous wave far-ultraviolet $(185 \mathrm{~nm})$ and pulsed laser (193 nm) radiation: XPS studies, J. Am. Chem. Soc., 1984, 106, 4288-4290.
37 R. Srinivasan and S. Lazare, Surface properties of poly(ethylene terephthalate) films modified by farultraviolet radiation at $193 \mathrm{~nm}$ (laser) and $185 \mathrm{~nm}$ (low intensity), J. Phys. Chem., 1986, 90, 2124-2131.

38 F. Chen, D. S. Zhang, Q. Yang, J. L. Yong, G. Q. Du, J. H. Si, F. Yun and X. Hou, Bioinspired Wetting Surface via Laser Micro-fabrication, ACS Appl. Mater. Interfaces, 2013, 5, 6777-6792.

39 L. Boinovich and A. Emelyanenko, A wetting experiment as a tool to study the physicochemical processes accompanying the contact of hydrophobic and superhydrophobic materials with aqueous media, Adv. Colloid Interface Sci., 2012, 179, 133-141.

40 R. R. Rye, J. A. Mann Jr and F. G. Yost, The Flow of Liquids in Surface Grooves, Langmuir, 1996, 12, 555-565.

41 K. Khare, S. Herminghaus, J. Baret, B. Law, M. Brinkmann and R. Seemann, Switching Liquid Morphologies on Linear Grooves, Langmuir, 2007, 23, 12997-13006.

42 J. Baret and M. Decre, Transport Dynamics in Open Microfluidic Grooves, Langmuir, 2007, 23, 5200-5204.

43 S. Burgess and T. Dawson, The Contribution of Fog to The Water Relations of Sequoia sempervirens (D. Don): Foliar Uptake and Prevention of Dehydration, Plant, Cell Environ., 2004, 27, 1023-1034.

44 Y. Xu, B. Yan and H. Zhu, Leaf Dew Contributes Nutrients to Paddies and Improves Rice Growth, Acta Agric. Scand., Sect. B, 2013, 63, 97-106. 